\title{
Submitted: 04.01.2020 \\ Accepted: \\ Sonoelastic response of median nerve to rehabilitation in carpal tunnel syndrome
} 26.02.2020

Published: 15.06.2020

\author{
Yasemin Durum Polat ${ }^{1}$, Elif Aydın², Fikriye Sinem Ince², Mehmet Bilgen ${ }^{3}$ \\ ${ }^{1}$ Department of Radiology, Aydin Adnan Menderes University, Faculty of Medicine, Aydin, \\ Turkey \\ ${ }^{2}$ Department of Physical Therapy and Rehabilitation, Aydn Adnan Menderes University, \\ Faculty of Medicine, Aydin, Turkey \\ ${ }^{3}$ Department of Biophysics, Aydin Adnan Menderes University, Faculty of Medicine, Aydin, Turkey \\ Correspondence: Assistant Prof. Dr. Yasemin Durum Polat, MD, Aydin Adnan Menderes \\ University, Faculty of Medicine, Department of Radiology, Aydın, Turkey; tel.: +905052291535, \\ fax:+92562146495, e-mail: yasemindurum@gmail.com
}

DOI: $10.15557 / J o U .2020 .0014$

Keywords

sonoelastography, ultrasonography, carpal tunnel syndrome, median nerve

\begin{abstract}
Aim of the study: To evaluate the sonoelastic response of the median nerve in patients with carpal tunnel syndrome following conservative rehabilitation with splint plus exercise regimens. Materials and methods: A total of thirty-five patients diagnosed with mild carpal tunnel syndrome and treated with splint plus exercise therapy; hand resting splint all day for 3 weeks and then only at nights along with nerve gliding exercises in 10 repetitions 3 times a day. The median nerve was evaluated clinically prior to the treatment and at week 6 of therapy using physical examination, electrodiagnostic neurophysiology tests and radiological imaging; Boston Scores, electromyogram, ultrasonography and sonoelastography. Results: Following the 6-week treatment protocol on 35 subjects with mild carpal tunnel syndrome, sonoelastography showed significantly softer median nerve, while the traditional parameters based on Boston Scores and cross-sectional area based on ultrasonography remained nearly unresponsive. Such early indication of biomechanical changes in the nerve may be of clinical importance if it can offer a prognostic value of the applied treatment, while tissue softening suggests the alleviation of nerve compression. Conclusions: Sonoelasticity of the median nerve can serve as a reliable marker for assessing therapeutic changes in median nerve stiffness and potentially the outcome early on in mild carpal tunnel syndrome.
\end{abstract}

\section{Introduction}

Carpal tunnel syndrome (CTS) is the most common type of neuropathy with a prevalence estimated at between $0.1-0.5 \%{ }^{(1)}$. The condition involves the entrapment of the median nerve under the flexor retinaculum, causing weakness, paresthesia and pain at the wrist and hand, and thus decreasing the quality of life. The severity of the disorder and effectiveness of any therapeutic intervention are typically determined by physical examination involving functional, performance and pain assessments, sensation and strength evaluations, and also by electrodiagnostic tests ${ }^{(2)}$.
However, such procedures are either painful or yield false negatives during the initial diagnosis or the following therapeutic period.

Ultrasonography (US) has been suggested as a non-invasive and inexpensive tool for the visualization of the entire median nerve and its surroundings ${ }^{(3)}$. Past data supports the utilization of US not only in the initial diagnosis of CTS, but also in determining the efficacy of applied therapy ${ }^{(4-6)}$. The cross-sectional area (CSA) of the median nerve within the carpal tunnel inlet and outlet is the parameter of interest measured to reflect the severity of CTS $^{(7)}$. As an 
extension to conventional US, sonoelastography (SE) has been introduced to map the local distribution of elasticity in biological tissues ${ }^{(8,9)}$.

Elasticity is defined as the ability of an object to maintain its original size and shape after being stretched or compressed $^{(10)}$. Pathologically abnormal tissue exhibits different elastic properties than normative characteristics ${ }^{(11)}$. Sonoelastic properties of peripheral nerves and other tissues have been investigated in the past ${ }^{(8,10,12-14)}$. In a limited number of papers, SE has been successfully utilized for the diagnosis of CTS, and the median nerve in patients with CTS has been reported to be stiffer than in healthy individuals $^{(10,13-15)}$. The beneficial utility of SE as a new way of assessing the therapeutic outcome has also been demonstrated after steroid injection into the carpal tunnel ${ }^{(3,15)}$ or following the release of the flexor retinaculum ${ }^{(16)}$.

At early stages, conservative approaches in the form of splinting and nerve gliding exercises are the main therapeutic strategies applied in patients with mild CTS. To the best of our knowledge, changes in the sonoelastic property of the median nerve after such combination therapy are yet to be examined. Therefore, with assessments based on SE imaging, this study primarily aims to evaluate the sonoelastic response of the median nerve in patients with CTS subjected to splinting plus exercise regimens. It also determines whether SE performed early on would offer any benefit in the clinical prognosis following therapy instituted for CTS.

\section{Materials and methods}

\section{Participants}

The study was approved by the institutional ethics committee (protocol no.: 23/12/2016-E.56735). An informed consent was obtained from all participants. The demographic variables of the patients such as age, weight, and symptom duration were recorded. The clinical diagnosis of CTS was based on the signs and recordings from confirmative electrophysiological measurements ${ }^{(17)}$. Nerve conduction was measured with electromyography using Nihon Kohden Neuropack-S1 (Tokyo-Japan). The severity of the disease was ranked using the GEHS Neurophysiological Classification System ${ }^{(18)}$. Patients categorized at stage 1, 2, and 3 were included in the study, as they were ideal candidates for conservative therapy. The exclusion criteria included previous surgery for CTS, injection to the carpal tunnel, pregnancy, polyneuropathy, and conditions that can cause CTS including diabetes mellitus, chronic inflammatory disease or thyroid disease.

Thirty-five patients with CTS were eligible and recruited into the cohort. The study was designed as a cross-over, as the same patient was examined repetitively; initially and at 6 weeks of therapy. The assessments at pre-therapy constituted a baseline reference for comparison against the readings of post-therapy. Boston Questionnaire was presented to all patients, consisting of two parts: symptom severity scale (Boston-S) and functional status scale (Boston-F). Their sum produced Boston total score (Boston-T). Higher scores indicated worse symptoms and degradation of the functional status ${ }^{(19)}$.

The patients were instructed to use a hand resting splint all day for 3 weeks, and then only at night. In addition, they were trained in home exercises for median nerve gliding, and performed the exercises in 10 repetitions and 3 times a day.

\section{Sonoelastography procedure}

The recruited patients with mild CTS were evaluated with free-hand SE under periodic recycling by a radiologist (YDP) working in the field for 7 years. The median nerve was imaged using 7-12 MHz linear array probe attached to Aplio 500 (Toshiba, Japan) ultrasound scanner. The scans were carried out during the afternoon hours at the beginning of the therapy and also at the end with a follow-up appointment. The patient was seated, and his or her hand with CTS was placed supine over a hard platform, while the elbow was kept at an angle of 90 degrees. The carpal tunnel was scanned in the transverse plane at the scaphoidpisiform level. The median nerve was identified by its gray scale contrast and echogenicity. The outline border of the nerve was manually drawn to measure the cross-sectional area CSA (Fig. 1). Then, SE scans were performed in realtime using a padding slab inserted between the probe and skin surface. The slab served as a pad made of a polymer with a fixed value of elasticity and acoustic characteristics similar to biological tissues. The polymer slab and the underlying carpal tunnel were deformed by compressiondecompression cycles via pressing the probe with force and frequency adjusted to an appropriate range according to the strain indicator depicted on the screen. Both static and dynamic images were acquired optimally, and recorded for further analysis. In the images, one region of interest (ROI) was placed over the median nerve, and another one of similar size over the slab (Fig. 2). The stiffness of the median nerve was assessed by strain index (SI) that was internally calculated by the built-in software of the scanner as the ratio of the longitudinal strains averaged over



Fig. 1. Transverse view of the median nerve on conventional B-mode ultrasonography as acquired from a 45-year-old patient. ROI was drawn manually. The area of the median nerve (CSA) was measured as $0.10 \mathrm{~cm}^{2}$ 


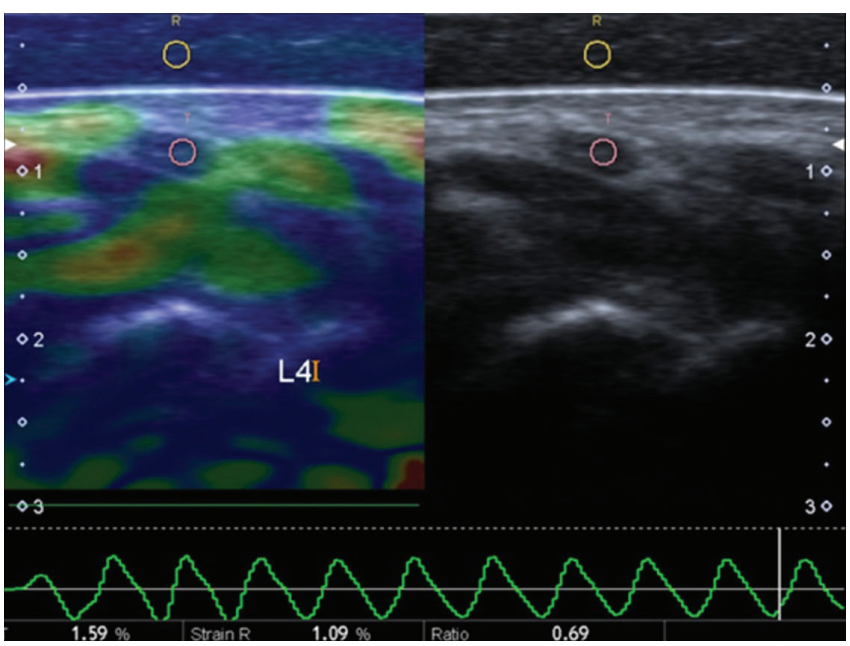

Fig. 2. Transverse SE image of a median nerve acquired from a 50-year-old female with mild CTS. SE was performed at the scaphoid-pisiform level. Circular ROIs were placed manually

the ROIs selected within the slab pad (SP) and the median nerve $(\mathrm{MN})$, and automatically shown on the screen. The SI measurements were repeated 5 times at different decompression phases, and the resultant average was tabulated for each individual patient.

\section{Statistical analysis}

The statistical software package SPSS Statistics for Windows Version 17 (SPSS Inc., Chicago, IL, USA) was used for all statistical analyses. The tabulated data involved parameters measured pre- and posttherapy: Boston Questionnaire, and CSA and SI measurements. The Kolmogorov-Smirnov test was used to evaluate whether the distributions of the measurements were normal. Comparisons between the groups were performed using Student's t-test. One-tailed paired Student's t-test was used to compare the data before and after the therapy. $P$-value of less than 0.05 was considered as indicating that the difference was statistically significant.

\section{Results}

The study subjects were aged $49.9 \pm 10.5$ years, and had BMI of $29.6 \pm 5.2$. With respect to gender distribution, the group included 30 females $(85.7 \%)$ and 5 males $(14.3 \%)$. SE was performed on a total of 61 wrists. Of the patients, dominant hand involvement was determined in $85.7 \%$, and symptom duration was longer than a year in $80 \%$. GEHS classification indicated $5.7 \%$ of patients as stage $1,31.4 \%$ as stage 2 and $62.9 \%$ as stage 3 .

The data collected during the study are summarized in Tab. 1. Boston scores assessed pre- and post-therapy are also presented in Fig. 3. Functional and Total scores did not show an improvement with the splint plus exercise therapy over time. However, the patients appeared to benefit from this combination therapy, as the difference between the Severity scores was statistically significant. The comparisons of the measurements based on the US and SE images of the median nerve are summarized in Fig. 4 and Fig. 5. According to the data, the applied therapy did not produce significant changes in the cross-sectional dimension of the median nerve, but made its sonoelastic property relatively softer.

\section{Discussions}

The present study was undertaken to examine the median nerve stiffness when CTS was treated with conservative approaches and the evaluation was based on SE measurements. The underlying pathophysiology of CTS is believed to be due to an increased pressure on the median nerve, which leads to ischemic injury and focal demyelination followed by fibrotic tissue deposition ${ }^{(20-22)}$. Epineural fibrosis under chronic compression is the main reason for stiffer nerve in patients with CTS. Increased stiffness decreases the nerve's ability to return to its original shape when compressed. Tendon and nerve gliding exercises and splints are conservatively recommended for cases of mild and moderate CTS ${ }^{(23)}$. Exercise with active wrist and finger motions and resting in splint improve venous return within the median nerve bundles, and subsequently decrease the pressure on the carpal tunnel ${ }^{(24)}$. The therapy ultimately reduces edema and indirectly prevents irreversible nerve damage.

In the diagnosis of CTS, gray scale US has widely been utilized, but attempts at using high-resolution magnetic resonance imaging also exist $\mathrm{t}^{(25,26)}$. Studies reported CSA of the median nerve ranging between 0.9 and $0.12 \mathrm{~cm}^{2(27-29)}$. In accordance, the measured CSA of the median nerve with CTS was about $0.10 \mathrm{~cm}^{2}$, and it was not responsive to the applied therapy (Fig. 4). Although the treatment strategy is different in principle when corticosteroid is locally injected to the carpal tunnel, the nerve area exhibited a slight increase in mild cases, but a significant decrement in moderate $\operatorname{cases}^{(3)}$.

We expected CSA would decrease after the therapy because edema would regress, but this did not happen. Our data indicated softening of the median nerve after 6 weeks of rehabilitation (Fig. 5). This confirmed

Tab. 1. Pre- and post-therapy assessments from a total of 35 patients

\begin{tabular}{|l|c|c|c|}
\cline { 2 - 4 } \multicolumn{1}{c|}{} & $\begin{array}{c}\text { Baseline } \\
\text { Median (25\%,75\%) }\end{array}$ & $\begin{array}{c}\text { Splint + Exercise } \\
\text { Median (25\%,75\%) }\end{array}$ & $p$ \\
\hline Boston-S & $31.50(27.00,40.00)$ & $30.00(22.25,36.50)$ & $\mathbf{0 . 0 4}$ \\
\hline Boston-F & $23.00(14.25,26.50)$ & $24.00(11.75,31.50)$ & 0.19 \\
\hline Boston-T & $50.50(45.00,66.75)$ & $40.25(53.00,69.25)$ & 0.32 \\
\hline CSA $\left(\mathrm{cm}^{2}\right)$ & $0.11(0.08,0.13)$ & $0.10(0.08,0.13)$ & 0.11 \\
\hline SP/MN SI & $0.83(0.33,1.66)$ & $0.69(0.31,1.17)$ & $\mathbf{0 . 0 3}$ \\
\hline
\end{tabular}

Boston carpal tunnel questionnaire; $S$ - symptom severity scale;

$\mathrm{F}$ - functional status scale; T - total; CSA - cross-sectional area;

SP/MN SI - slab pad/median nerve strain index 


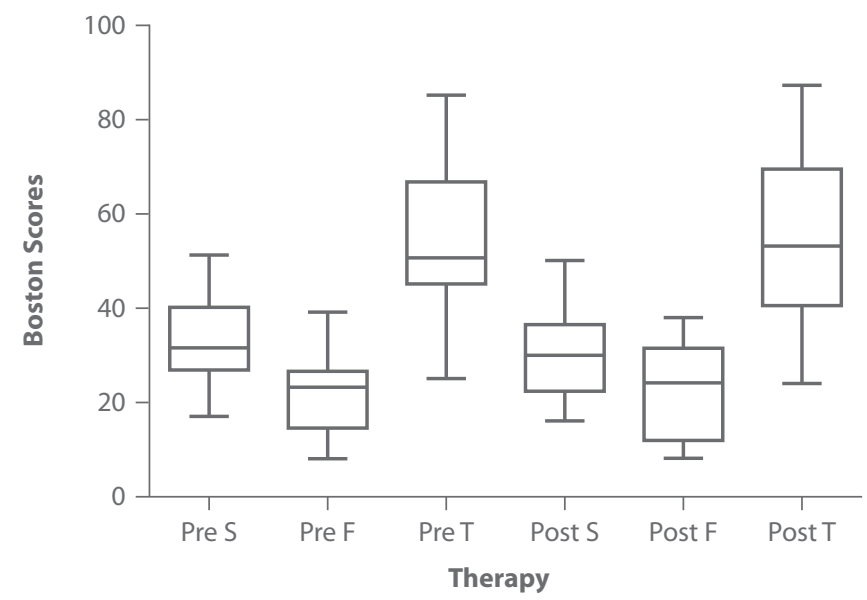

Fig. 3. Boston Scores pre- and post-therapy. The difference between Pre- and Post $S$ is statistically significant $(p<0.05)$, but $F$ and $T$ are not $(p>0.05)$

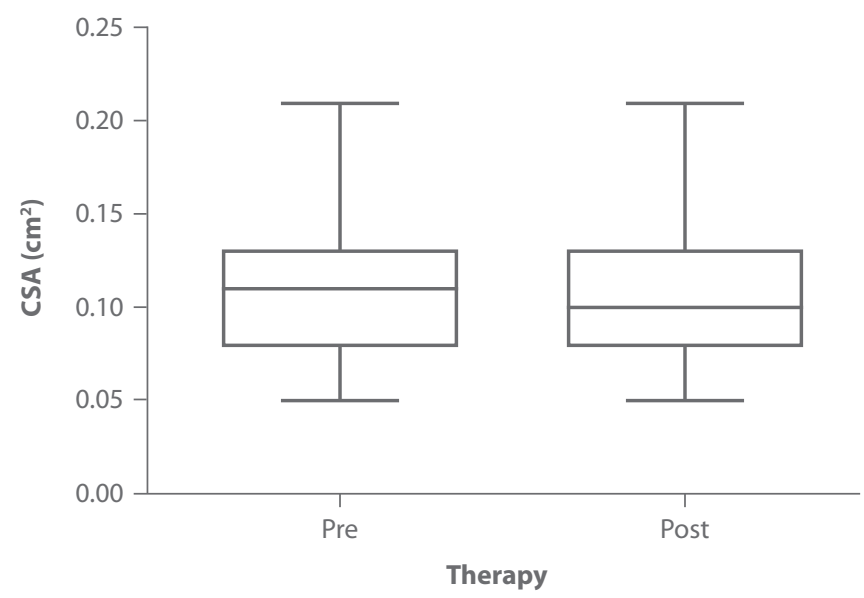

Fig. 4. Cross-sectional area (CSA) of the median nerves pre- and post-therapy. The difference is statistically insignificant ( $p>0.05)$



Fig. 5. Measurements of strain index (SI) pre- and post-therapy. The difference is statistically significant $(p<0.05)$ that the therapeutic outcome of CTS could be evaluated independently with SE, as validated earlier under other conditions by other researchers ${ }^{(3,15,16)}$. Tissue softening may have clinical implications, as it may be indicative of the beneficial consequences of the rehabilitation process. More importantly, it raises the important question of how exercise in conjunction with splint changes the biomechanical properties of the median nerve, while its CSA is preserved. We could explain this finding by the ongoing pathological changes leading to structural remodeling, such as internal fibrosis or scar tissue formation within the nerve ${ }^{(30)}$. Based on our data, tissue softening arises from further prevention of the formation of epineural fibrosis by the gliding exercise rather than the splint activity alone. The possibility of inducing biomechanical changes via external events has important clinical implications, as tissue softening is naturally expected to reduce the pressure on the nerve, and this effect would ultimately improve recovery from CTS. However, functional improvement was unfortunately not confirmed by other parameters (CSA and Boston scales), and thus stands out as an enigma. Clinical response to the therapy may be developing at a slower pace, and performing the evaluation at 6 weeks may not be a sufficient enough time frame.

The study had its limitations. Firstly, a single radiologist performed the US evaluations and thus inter-observer variability could not be investigated. Similar studies performed previously indicated strong inter-observer variability for elastography, so we would expect the same finding if this study had been done in the same manner ${ }^{(3,15,16)}$. Secondly, we evaluated median nerve stiffness but not carpal tunnel stiffness because our rehabilitation protocol involved median nerve gliding exercise. Increased carpal tunnel pressure affects not only the nerve but also the tendons. Previous literature has revealed significant changes in the stiffness of the carpal tunnel rather than the median nerve ${ }^{(3,15)}$. Thirdly, follow-up evaluations at further time points were not performed, as the study was a pilot project to explore the feasibility and merits of SE in the therapy of CTS.

\section{Conclusion}

In conclusion, $\mathrm{SE}$ is capable of sensing tissue stiffness, and it provides information on changes in the biomechanical properties of the median nerve. SE-based measurements can serve as an early indicator for evaluating the response of the conservative splint plus exercise therapy in CTS than traditional functional and symptomatic characterization of recovery.

\section{Conflict of interest}

The authors do not report any financial or personal connections with other persons or organizations, which might negatively affect the contents of this publication and/or claim authorship rights to this publication 


\section{References}

1. Stevens JC: AAEM minimonograph \#26: the electrodiagnosis of carpal tunnel syndrome. American Association of Electrodiagnostic Medicine. Muscle Nerve 1997; 20: 1477-1486.

2. Lim YH, Chee DY, Girdler S, Lee HC: Median nerve mobilization techniques in the treatment of carpal tunnel syndrome: A systematic review. J Hand Ther 2017; 30: 397-406.

3. Asadov R, Erdal A, Buğdaycı O, Gündüz OH, Ekinci G: The effectiveness of ultrasonography and ultrasonographic elastography in the diagnosis of carpal tunnel syndrome and evaluation of treatment response after steroid injection. Eur J Radiol 2018; 108: 172-176.

4. Mondelli M, Filippou G, Aretini A, Frediani B, Reale F: Ultrasonography before and after surgery in carpal tunnel syndrome and relationship with clinical and electrophysiological findings. A new outcome predictor? Scand J Rheumatol 2008; 37: 219-224.

5. Smidt MH, Visser LH: Carpal tunnel syndrome: clinical and sonographic follow-up after surgery. Muscle Nerve 2008; 38: 987-991.

6. Vögelin E, Nüesch E, Jüni P, Reichenbach S, Eser P, Ziswiler HR: Sonographic follow-up of patients with carpal tunnel syndrome undergoing surgical or nonsurgical treatment: prospective cohort study. J Hand Surg Am 2010; 35: 1401-1409.

7. Zhang L, Rehemutula A, Peng F, Yu C, Wang TB, Chen L: Does the ratio of the carpal tunnel inlet and outlet cross-sectional areas in the median nerve reflect carpal tunnel syndrome severity? Neural Regen Res 2015; 10: 1172-1176.

8. Karaman CZ, Başak S, Polat YD, Ünsal A, Taşkın F, Kaya E et al.: The role of real-time elastography in the differential diagnosis of salivary gland tumors. J Ultrasound Med 2019; 38: 1677-1683.

9. Bilgen M: Target detectability in acoustic elastography. IEEE Trans U1trason Ferroelectr Freq Control 1999; 46: 1128-1133.

10. Orman G, Ozben S, Huseyinoglu N, Duymus M, Orman KG: Ultrasound elastographic evaluation in the diagnosis of carpal tunnel syndrome: initial findings. Ultrasound Med Biol 2013; 39: 1184-1189.

11. Bilgen M, Insana MF: Elastostatics of a spherical inclusion in homogeneous biological media. Phys Med Biol 1998; 43: 1-20.

12. Ning CP, Jiang SQ, Zhang T, Sun LT, Liu YJ, Tian JW: The value of strain ratio in differential diagnosis of thyroid solid nodules. Eur J Radiol 2012; 81: 286-291.

13. Ghajarzadeh M, Dadgostar M, Sarraf P, Emami-Razavi SZ, Miri S, Malek M: Application of ultrasound elastography for determining carpal tunnel syndrome severity. Jpn J Radiol 2015; 33: 273-278.

14. Kantarci F, Ustabasioglu FE, Delil S, Olgun DC, Korkmazer B, Dikici AS et al.: Median nerve stiffness measurement by shear wave elastography: a potential sonographic method in the diagnosis of carpal tunnel syndrome. Eur Radiol 2014; 24: 434-440.

15. Miyamoto H, Halpern EJ, Kastlunger M, Gabl M, Arora R, BellmannWeiler R et al.: Carpal tunnel syndrome: diagnosis by means of median nerve elasticity-improved diagnostic accuracy of US with sonoelastography. Radiology 2014; 270: 481-486.

16. Yoshii Y, Ishii T, Etou F, Sakai S, Tanaka T, Ochiai N: Reliability of automatic vibratory equipment for ultrasonic strain measurement of the median nerve. Ultrasound Med Biol 2014; 40: 2352-2357.

17. Beekman R, Visser LH: Sonography in the diagnosis of carpal tunnel syndrome: a critical review of the literature. Muscle Nerve 2003; 27: 26-33.

18. Greathouse DG, Ernst G, Halle JS, Shaffer SW: GEHS neurophysiological classification system for patients with carpal tunnel syndrome. US Army Med Dep J 2016: 60-67.

19. Levine DW, Simmons BP, Koris MJ, Daltroy LH, Hohl GG, Fossel AH et al.: A self-administered questionnaire for the assessment of severity of symptoms and functional status in carpal tunnel syndrome. J Bone Joint Surg Am 1993; 75: 1585-1592.

20. Mackinnon SE: Pathophysiology of nerve compression. Hand Clin 2002; 18: 231-241.

21. Ibrahim I, Khan WS, Goddard N, Smitham P: Carpal tunnel syndrome: a review of the recent literature. Open Orthop J 2012; 6: 69-76.

22. Rempel D, Dahlin L, Lundborg G: Pathophysiology of nerve compression syndromes: response of peripheral nerves to loading. J Bone Joint Surg Am 1999; 81: 1600-1610.

23. Baysal O, Altay Z, Ozcan C, Ertem K, Yologlu S, Kayhan A: Comparison of three conservative treatment protocols in carpal tunnel syndrome. Int J Clin Pract 2006; 60: 820-828.

24. Seradge H, Bear C, Bithell D: Preventing carpal tunnel syndrome and cumulative trauma disorder: effect of carpal tunnel decompression excercises: an Oklahoma experience. J Okla State Med Assoc 2000; 93: 150-153.

25. Bilgen M, Heddings A, Al-Hafez B, Hasan W, McIff T, Toby B et al.: Microneurography of human median nerve. J Magn Reson Imaging 2005; $21: 826-830$.

26. Heddings A, Bilgen M, Nudo R, Toby B, McIff T, Brooks W: High-resolution magnetic resonance imaging of the human median nerve. Neurorehabil Neural Repair 2004; 18: 80-87.

27. Mallouhi A, Pülzl P, Trieb T, Piza H, Bodner G: Predictors of carpal tunnel syndrome: accuracy of gray-scale and color Doppler sonography. AJR Am J Roentgenol 2006; 186: 1240-1245.

28. Wiesler ER, Chloros GD, Cartwright MS, Smith BP, Rushing J, Walker FO: The use of diagnostic ultrasound in carpal tunnel syndrome. J Hand Surg Am 2006; 31: 726-732.

29. Roll SC, Evans KD, Li X, Freimer M, Sommerich CM: Screening for carpal tunnel syndrome using sonography. J Ultrasound Med 2011; 30: 1657-1667.

30. Rempel DM, Diao E: Entrapment neuropathies: pathophysiology and pathogenesis. J Electromyogr Kinesiol 2004; 14: 71-75. 